\title{
Galactic winds and the symmetry properties of galactic magnetic fields
}

\author{
D. Moss ${ }^{1}$, D. Sokoloff ${ }^{2}$, R. Beck ${ }^{3}$, and M. Krause ${ }^{3}$ \\ 1 School of Mathematics, University of Manchester, Oxford Road, Manchester, M13 9PL, UK \\ e-mail: moss@ma.man.ac.uk \\ 2 Department of Physics, Moscow University, 119992 Moscow, Russia \\ 3 Max-Planck-Institut für Radioastronomie, Auf dem Hügel 69, 53121 Bonn, Germany \\ Received 20 October 2009 / Accepted 26 January 2010
}

ABSTRACT

\begin{abstract}
Context. Contemporary studies of the symmetry properties of galactic magnetic fields contain two contradictory ideas. There are observational and theoretical hints that large-scale magnetic fields are symmetric with respect to the galactic plane in the disc, and antisymmetric in the halo. On the other hand, standard galactic dynamo models without a galactic wind give symmetric or antisymmetric configurations in both disc and halo regions simultaneously.

Aims. Here we explore the role of a galactic wind in resolving this dichotomy.

Methods. The key idea is that a galactic wind must be be taken into account in order to resolve the problem. We perform a search of the parameter space of galactic dynamo models containing such winds.

Results. By including a galactic wind, magnetic configurations of such symmetry, i.e. approximately even in the disc and odd in the halo, can be obtained. We note that a variety of other solutions can also be found.

Conclusions. A galactic wind plays a significant role in determining the structure of galactic magnetic configurations and should be included in future modelling.
\end{abstract}

Key words. magnetohydrodynamics - galaxies: magnetic fields - galaxies: spiral - galaxies: halos - ISM: magnetic fields

\section{Introduction}

Based on the mathematical properties of thin disc dynamos (e.g Ruzmaikin 1988), and supported by most of the available observational evidence (e.g. Krause 1989; Beck 2009; Heesen et al. 2009b), until quite recently there has been a rather general belief that the magnetic fields throughout the disc and halo of spiral galaxies are of predominantly even (quadrupole-like) symmetry. This conclusion has been supported by a number of numerical investigations of "disc + halo" models (e.g. Brandenburg et al. 1992; Elstner et al. 1992; Brandenburg et al. 1993; Beck et al. 1996), in which the magnetic field was generated largely or wholely in the disc. However such a conclusion certainly is not inevitable from a theoretical viewpoint, and as early as 1990 Sokoloff \& Shukurov (1990) suggested that the quasi-spherical halo region might support a quasi-independent dynamo with odd parity field, and thus that disc and halo fields might have different parity properties. There is also the consequence that the halo field might be oscillatory, but given the probable periods - of order Gyr - there would be no direct observational consequences of periodicity.

It should be noted that observational information on largescale magnetic fields comes from polarized radio emission and Faraday rotation measures (RM) (Beck 2009). The patterns of B-vectors of polarized emission generally indicate global fields. Measurements of the field parity needs Faraday rotation data from polarization observations at several frequencies. However, polarized emission is weak and affected by frequency-dependent Faraday depolarization, so that the accuracy of RM data is low. RM of polarized background sources can yield higher accuracy, but such sources are rare. Furthermore, information concerning the disc component of the magnetic field in general comes from more or less face-on galaxies while that concerning the halo component comes from edge-on galaxies, i.e. comprehensive information from a single object is hard to obtain and is available up to now only for two galaxies: NGC 253 and the Milky Way, which will be described below.

Recent progress in the observation and interpretation of polarized emission has allowed the separation of the contributions from disc and halo regions. Studying polarized emission and rotation measures from background sources, Sun et al. (2008) came to the conclusion that the Milky Way hosts a large-scale magnetic field in the halo, with a reversal between the field above and below the disc (odd parity), whereas the disc field has the same direction above and below the galactic equator (even parity). This appears to be consistent with the suggestion of Sokoloff \& Shukurov (1990) concerning different symmetries in disc and halo magnetic field components.

Furthermore, there is increasing evidence that the Milky Way drives a strong galactic wind which removes angular momentum at a considerable rate. The model of a wind of hot gas driven by cosmic rays and thermal gas by Everett et al. (2008) can explain the diffuse soft X-ray emission. According to this model, the initial wind velocity of about $200 \mathrm{~km} \mathrm{~s}^{-1}$ increases to about $600 \mathrm{~km} \mathrm{~s}^{-1}$ at $10 \mathrm{kpc}$ height, and the mass loss rate is about 2 solar masses per year.

In external galaxies, the parity of the disc and halo magnetic field is much more difficult to determine due to the faint polarized radio emission of the halo and the lack of polarized background sources. Heesen et al. (2009a,b) presented radio continuum polarimetry observations of the nearby edge-on galaxy NGC 253 which possesses a very bright radio halo. Using 
the vertical synchrotron emission profiles and the lifetimes of cosmic-ray electrons, they determined the cosmic-ray bulk speed as $300 \pm 30 \mathrm{~km} \mathrm{~s}^{-1}$, indicating the presence of a strong galactic wind in this galaxy. The large-scale magnetic field was decomposed into a axisymmetric toroidal component in the disk and a poloidal component in the halo. The poloidal component shows a prominent $X$-shaped magnetic field structure centered on the nucleus, similar to the magnetic field observed in other edge-on galaxies (Krause 2009). Faraday rotation measures indicate that both the toroidal and the poloidal fields have an even parity (i.e. are symmetric with respect to the disc plane). However an odd parity poloidal field cannot be excluded, because the Faraday data for the halo are not conclusive. For all other external galaxies observed so far, the Faraday data do not allow any conclusion.

Summarizing, the interpretation of the observations of Sun et al. (2008) is that we see in the Milky Way a superposition of a conventional even-parity toroidal (disc) field and an odd-parity poloidal (halo) field, probably of X-shape as in external galaxies, i.e. a field with a vertical component and a radial component of similar strength. The observations of external galaxies also tell us that the ratio of strengths of the vertical to the radial component increases with height.

On the other hand, on reflection the argument given by Sokoloff \& Shukurov (1990) may not be convincing. Their argument is based on the geometrical similarity of the quasi-spherical gaseous disc halo with the solar convective spherical shell. The latter excites a magnetic field of dipole symmetry. The point is however that the magnetic link between the two hemispheres of a quasi-spherical dynamo operating in the galactic halo is not very strong. The presence of a disc component separating the two hemispheres makes the magnetic link between the hemispheres even smaller, and the situation even less predictable.

Moss \& Sokoloff (2008) made a study of more-or-less conventional galactic dynamo models, while allowing the presence of significant dynamo action in the halo. Their results largely supported the conventional viewpoint concerning the coexistence of magnetic fields of different symmetries in the galactic disc and halo. Moss \& Sokoloff (2008) demonstrated that usually such a "two-part" dynamo generates either a quadrupole-like or a dipole-like magnetic field both in the disc and in the halo, i.e. either the disc enslaves the halo or vice versa. There are some exceptional cases with mixed parity solutions, but these appear insufficient to explain the observations.

Thus there appears now to be a possible contradiction between the predictions of galactic dynamo theory and the observational results. This requires investigation and elucidation, and that is the aim of this paper.

We should emphasize from the beginning that we are going to consider generic models for galactic magnetic fields, assuming that the observed magnetic configuration of the Milky Way, for example, does not depend on peculiar details of its hydrodynamics. Thus, we believe that it is not fruitful to attempt to reproduce in fine detail the hydrodynamics of this, or any other, particular galaxy, which are in any case not known in full detail. Rather, we will attempt to isolate the decisive factor(s) leading to the observed magnetic field configuration. Our result is that a galactic wind can be such a factor.

\section{The galactic model}

We consider the magnetic field in galactic discs and halos to be generated by a standard mean-field dynamo based on differential rotation and the mirror asymmetry of galactic turbulence known as the $\alpha$-effect ( $\alpha \omega$-dynamo), and specify the dynamo action in

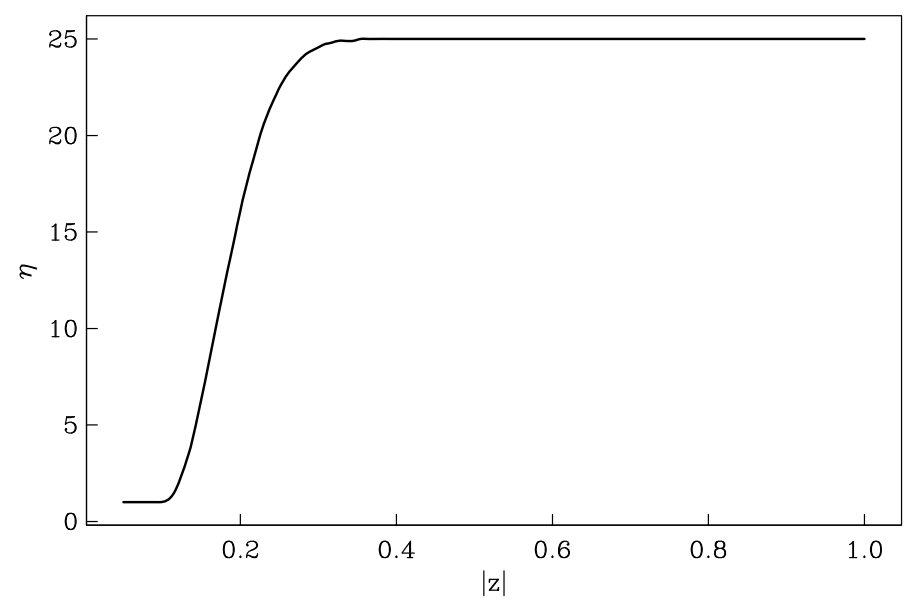

Fig. 1. The dependence of the diffusivity $\eta$ on $|z|$, the distance from the disc plane, for a diffusivity contrast of 25

disc and halo components using dynamo governing parameters chosen to the best of our knowledge of galactic hydrodynamics, and of the generic geometry of spiral galaxies. For the dynamo saturation mechanism, we use the simplest algebraic $\alpha$ quenching.

In particular, the rotation law is chosen as in Moss \& Sokoloff (2008) with the Brandt turnover radius 0.1R ( $R$ is the galactic radius, say $20 \mathrm{kpc})$. The turbulent diffusivity in the disc component is $\eta=\eta_{\text {disc }}$ in $|z|<z_{\mathrm{d}}=0.1 R$, where $z= \pm z_{\mathrm{d}}$ are upper and lower disc boundaries. In the halo $\left(|z|>z_{\mathrm{d}}\right)$,

$\eta=\eta_{\text {disc }}\left(1-\eta_{1} \exp \left(-\left(\left(z-z_{\mathrm{d}}\right) / z_{\mathrm{w}}\right)^{2}\right)\right) /\left(1-\eta_{1}\right)$

where $\eta_{1}=1-\eta_{\text {disc }} / \eta_{\text {halo }}$ and $z_{\mathrm{w}}$ stands for the spatial scale of the wind. We specify the diffusivity contrast $\eta_{\text {halo }} / \eta_{\text {disc }}=25$ and $z_{\mathrm{w}}=z_{\mathrm{d}}$. The first contrast is 5 times larger than that used by Moss \& Sokoloff (2008); this is technically possible because we choose here a smoother transition between disc and halo than in the previous paper. The dependence of $\eta$ on $z$ is shown in Fig. 1, and can be compared with that shown in Fig. 1 of Moss \& Sokoloff (2008). The wind velocity field is defined as follows: at first it is vertical $\left(|z|<z_{\mathrm{W}}\right)$, then becomes radial, the transition occurring smoothly over a length scale $z_{\mathrm{w}}$. More specifically, for

$z<z_{\mathrm{w}}, \boldsymbol{u}=u_{z} \hat{z}$, with $u_{z}=U_{0}\left(1-\exp \left(-z / z_{\mathrm{w}}\right)\right)$,

and in

$z>2 z_{\mathrm{w}}, \boldsymbol{u}=u_{r} \hat{\boldsymbol{r}}$, with $u_{r}=U_{0}\left(1-\exp \left(-z / z_{\mathrm{w}}\right)\right)$,

and in $z_{\mathrm{w}} \leq z \leq 2 z_{\mathrm{w}}$ there is a smooth interpolation. All scalings are performed in terms of $\eta_{\text {disc }}$ and $R$. Thus the dimensionless dynamo governing parameters responsible for the amplitudes of wind, differential rotation and $\alpha$-effect in disc and halo are correspondingly $C_{\text {wind }}=U_{0} R / \eta_{\text {disc }}\left(U_{0}\right.$ is the wind amplitude), $R_{\omega}=\Omega_{0} R^{2} / \eta_{\text {disc }}\left(\Omega_{0}\right.$ is as appears in the Brandt law $)$, $R_{\alpha, \text { disc }}=\alpha_{0, \text { disc }} R / \eta_{\text {disc }}$ and $R_{\alpha \text {, halo }}=\alpha_{0 \text {, halo }} R / \eta_{\text {disc }}$ (subscript zero on alpha denotes the unquenched values). The spatial distribution of $\alpha$ is as in Moss \& Sokoloff (2008).

We fully appreciate that the model is oversimplified and does not take into account many of the finer details of galactic hydrodynamics. Also it ignores recent trends in galactic dynamo theories, such as dynamical schemes for dynamo saturation based on magnetic helicity conservation (e.g. Kleeorin et al. 2002). In principle, it would be no problem to include many such effects in the model, provided particular values for specific quantities 
responsible for the effects were known for the galaxy under consideration. We avoid this level of sophistication because we are attempting to isolate a possible generic explanation of the observational result. We note, in passing, that Kleeorin et al. (2003) produce some evidence to suggest that results from such an approach may not differ widely from those of a naive model.

The key ingredient of the model is the galactic wind which is traced by the cosmic-ray bulk flow, which is as large as $u=300 \pm 30 \mathrm{~km} \mathrm{~s}^{-1}$ (Heesen et al. 2009a). Heesen et al. argue that the cosmic-ray bulk flow is the sum of the wind velocity of the gas and Alfvén velocity, which may reduce the estimate by up to $150 \mathrm{~km} \mathrm{~s}^{-1}$. We believe that it would be unwise to identify even this value directly as the mean velocity of galactic wind $U_{0}$ which governs the wind effect in the model (this speed can be compared to the rotation velocity of $250 \mathrm{~km} \mathrm{~s}^{-1}$ ). Our interpretation is that the observed value of $u$ corresponds to the velocity of a component that comprises a tiny mass fraction of the galactic medium, so that the averaged quantity $U_{0}$ is substantially smaller; we assume a few $\mathrm{km} \mathrm{s}^{-1}$. This is why we take $C_{\text {wind }}$, determined by $U_{0}$ (and given by the above definition, see also Sect. 4) as a free parameter, to be used in fitting the model to observations.

We use the code described in Moss \& Sokoloff (2009), which employs a 2nd order Runge-Kutta integrator on a finite difference grid. With $\eta_{\text {halo }} / \eta_{\text {disc }}=25$ the presence of the diamagnetic velocities $-\frac{1}{2} \nabla \eta$ requires for adequate accuracy a spatial resolution of $101 \times 201$ points, distributed uniformly in radius and polar angle $0 \leq \theta \leq \pi$ respectively. Accuracy was checked in a couple of cases at a resolution of $201 \times 401$ grid points - at most the differences in energy and parity were about $5 \%$, and the field geometry was not significantly altered.

\section{Exploring the parameter space}

We performed a range of numerical experiments, integrating the mean-field dynamo equations for our model in a parametric range which appears reasonable in the galactic context, as judged from our previous experiences of modelling (e.g. Brandenburg et al. 1992, 1993; Moss \& Sokoloff 2008). We present our results initially in the form of synoptic maps in the $\left(C_{\text {wind }}, R_{\alpha, \text { halo }}\right)$ plane, for $R_{\alpha \text {, disc }}=10$ (Fig. 2, upper panel) and $R_{\alpha \text {, disc }}=20$ (Fig. 2, lower panel). We adopt this approach because conventional ideas suggest that the plausible range of values for $R_{\alpha, \text { disc }}$ is rather small. For example, with $\alpha_{\text {disc }} \lesssim 1 \mathrm{~km} \mathrm{~s}^{-1}, \eta_{\text {disc }} \gtrsim 10^{26} \mathrm{~cm}^{2} \mathrm{~s}^{-1}$, we get $R_{\alpha, \text { disc }} \lesssim 50$.

We present results for a single value of $R_{\omega}=2 \times 10^{4}$. This is in part to simplify our discussion of a multi-parameter problem, but also because we feel that this quantity is determined better (accepting uncertainties in the value of the turbulent diffusivity) than others in the problem. We summarize the gross symmetry properties of our models with respect to the galactic plane by the quantity $P . P=+1$ corresponds to pure even (quadrupole-like) symmetry, $P=-1$ to the pure odd (dipole-like) case, and values $-1<P<+1$ to intermediate cases.

Our interpretation is that the models indicated by plus signs $\left(P_{\mathrm{h}}, P_{\mathrm{d}} \approx+1\right)$ show the models where the disc enslaves the halo, while the open diamonds in Fig. 2 show models where the halo component of field enslaves that of the disc. The former case corresponds to the conventional models discussed at the beginning of this paper. As expected, they occur in the region of moderate $R_{\alpha}$, halo values, i.e. with weak dynamo activity in the halo. Cases where the halo enslaves the disc (signs) are, as expected, rarer when $R_{\alpha \text {, disc }}$ is larger. Decaying magnetic fields occur when the
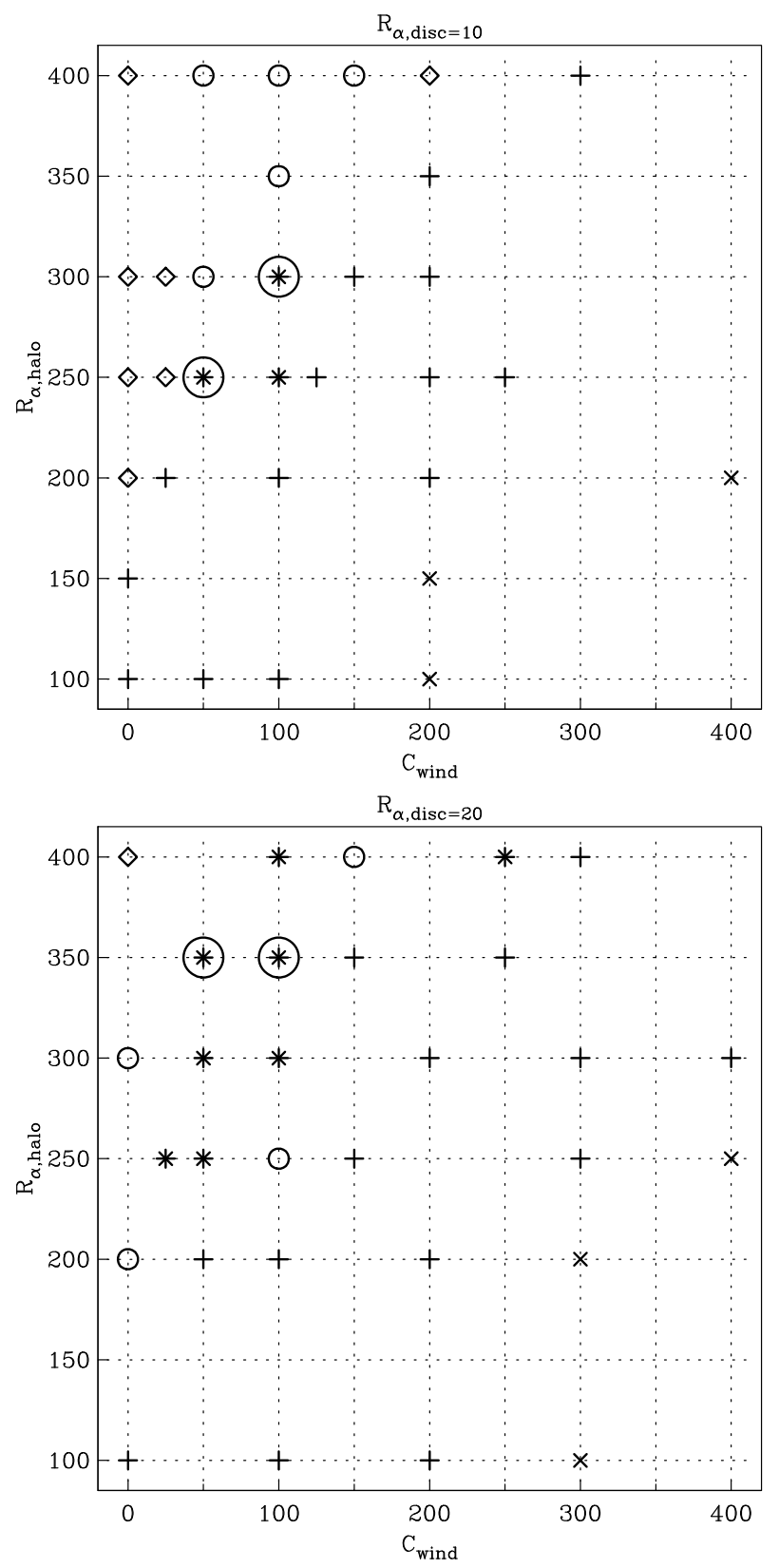

Fig. 2. Synoptic diagrams for the parities $P_{\mathrm{d}}, P_{\mathrm{h}}$ respectively of the disc and halo magnetic field components. The upper panel is for $R_{\alpha, \text { disc }}=10$ and the lower for $R_{\alpha, \text { disc }}=20$. In both panels, asterisks denote oscillating (at low amplitude) or steady models with $P_{\mathrm{d}} \approx+1, P_{\mathrm{h}} \approx-1-$ see discussion in the text. Open diamonds show the location of models with $P_{\mathrm{d}}, P_{\mathrm{h}} \approx-1$, plus signs those with $P_{\mathrm{h}}, P_{\mathrm{d}} \approx+1$. Open circles indicate examples with intermediate behaviour not included in the above categories, and open circles containing asterisks indicate oscillatory solutions which at times are in or near to a state corresponding to that indicated by an asterisk. Crosses show decaying solutions, where a dynamo does not operate.

wind is too strong and advects the dynamo generated magnetic field more rapidly than it is created. Understandably, the region in Figs. 2 occupied by such solutions shrinks as $R_{\alpha \text {, disc }}$ grows. Some models are strictly steady, but most oscillate at low amplitude (typically less than $10 \%$ in energy, smaller in parity). The properties of these two groups of solutions do not seem very different, and thus we do not distinguish them in Fig. 2. (The periods of the oscillatory models are far too long to be of 

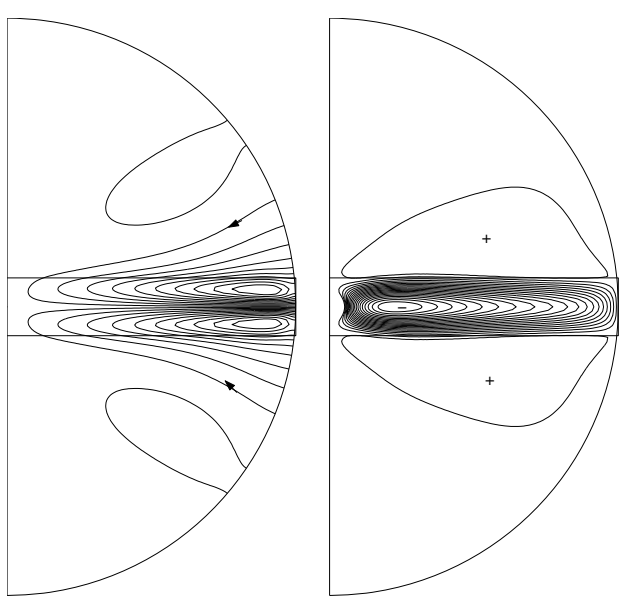

Fig. 3. Poloidal field lines (left hand panel), contours of toroidal field strength (right hand panel) in cuts through half a meridian plane for a model with $R_{\alpha, \text { disc }}=20, C_{\text {wind }}=0, R_{\alpha \text {, halo }}=100$. In the right hand panel regions of positive/negative toroidal field are indicated by $+/-$ signs respectively.

observational interest!) The occurrence of various intermediate cases also appears understandable.

For orientation, we show in Fig. 3 the field structure of a more-or-less conventional galactic dynamo model with $C_{\text {wind }}=$ 0 and a relatively small value of $R_{\alpha, \text { halo. However our main }}$ interest is with the cases indicated by asterisks in Fig. 2, i.e. combinations of approximately quadrupole-like fields in the disc and approximately dipole-like fields in the halo, as indicated by the observational data in the Milky Way ${ }^{1}$. These models often are oscillatory, in the halo at least. We consider fields to be quadrupole-like in the disc if $P_{\text {disc }}>+0.8$, and dipole-like in the halo if $P_{\text {halo }}<-0.8$ - such solutions would appear observationally as having quadrupolar/dipolar fields respectively. In most such cases, $P_{\text {halo }}$ is very near -1 . Solutions that are, for part of the time only, in or near to a state that could be denoted by an asterisk are shown by an asterisk within a circle. The existence of this configuration confirms in some (weak) sense the concept suggested by Sokoloff \& Shukurov (1990). We stress however that asterisks (and other symbols) in Fig. 2 describe only the symmetry of the solutions, and that further description of the magnetic configuration is required. The area in the parameter space at the upper left corner of the diagrams where asterisks occur is the region in which we find models that can hope to explain the observations of the Milky Way. We note that for $R_{\alpha, \text { disc }}=10$ this region is separated from the vertical axis, so that such a configuration requires significant wind.

We note a significant difference between the models of this paper and those of Moss \& Sokoloff (2008). Here we take a larger contrast in turbulent diffusivity between disc and halo (25), with a smoother transition between these regions, whereas Moss \& Sokoloff (2008) took a lower diffusivity contrast $(\leq 5)$ with a much more abrupt transition. Some experiments with the present $\eta$ profile and $\eta_{\text {halo }} / \eta_{\text {disc }}=5$ showed that a similar range of behaviour was found as with the larger diffusivity contrast, and again that with the larger value of the disc dynamo number $R_{\alpha, \mathrm{d}}$ it was perhaps a little easier to find solutions of the type denoted by an asterisk.

Magnetic configurations in the area occupied by asterisks can have poloidal field lines in the halo which resemble X-like

\footnotetext{
1 Whereas NGC 253 may correspond to the "conventional" model, as indicated by plus signs in Fig. 2.
}

structures, to various degrees of conviction, as can certain other fields with non-quadrupole-like fields in the halo. We consider fields to have X-like structures when a substantial part of the poloidal field is quasi-radial. We show in the left hand panels of Figs. 3 and 4 four poloidal field structures. For $R_{\alpha \text {, disc }}=20$, $C_{\text {wind }}=0$ and the relatively small $R_{\alpha \text {, halo }}=100$ we obtain something like the traditional galactic dynamo model without wind or disc dynamo component (Fig. 3). In both this case and that shown in row (c) of Fig. 4, the poloidal field is far from $\mathrm{X}$-shaped. On the other hand we consider the fields shown in the left hand panels of rows (a) and (b) to be approximately X-like.

The incidence in parameter space of X-like fields seems to follow a certain pattern. Fields of approximately odd parity in the halo are rather X-like - this structure is close to the natural configuration of a dipolar field away from the equator. This tendency is reinforced by the presence of a relatively weak wind. However, as the wind gets stronger, the disc-halo linkage gets stronger and eventually there is a tendency for both the disc and halo fields to have even parity, plausibly as the influence of the disc is advected into the halo. Such fields are, for the parameters we investigated, inherently even in the presence of a wind. The change in field geometry as the wind strength increases at given $R_{\alpha, \text { disc }}$ can be seen in Fig. 4 .

\section{Discussion and conclusions}

Summarizing the results, we conclude that the physical processes incorporated into standard galactic dynamo theory are sufficient to reproduce the generic properties of the magnetic field of both the Milky Way and NGC 253. We note here that there are still various problems with the comparison of observations of polarized emission from disc and halo of a given galaxy and theoretical models of magnetic field generation. In particular, a separation of magnetic field into poloidal and toroidal components is straightforward in theoretical studies, whereas from the observational viewpoint it requires a sophisticated interpretation of patterns of polarized emission. We appreciate that much remains to be done to elaborate the finer details of such a comparison.

We should acknowledge here that Brandenburg et al. (1993) found $X$-like magnetic fields when simulating magnetic fields in galactic halos in the presence of a galactic wind. However their models all had even parity (quadrupole-like) magnetic fields throughout. At that time observations could not give sufficient information about field parities for the importance of the issue to be realized, and so the question of quasi-independent dynamo action in disc and halo was not pursued.

We note that the topic under discussion is a challenge for galactic dynamo theory. Dealing with disc and halo components of magnetic field taken separately, we have a firm basis (see e.g. Beck et al. 1996) for relatively simple theoretical concepts that can explain the observations, at least at a qualitative level. In contrast, although we have here been able to present dynamo models which can explain the coexisting magnetic structures in disc and halo, we have severe difficulties in predicting which set of dynamo governing parameters is most appropriate to explain a given configuration. We believe that further development of qualitative methods of galactic dynamo theory in this direction is required.

An even more substantial shortcoming of galactic dynamo theory illuminated by our studies is connected with the multiphase nature of the galactic medium. We recall that a straightforward estimate of the wind velocity is $\geq 150 \mathrm{~km}^{-1}$ (Heesen et al. 2009a), but this flow is associated with only a small part 


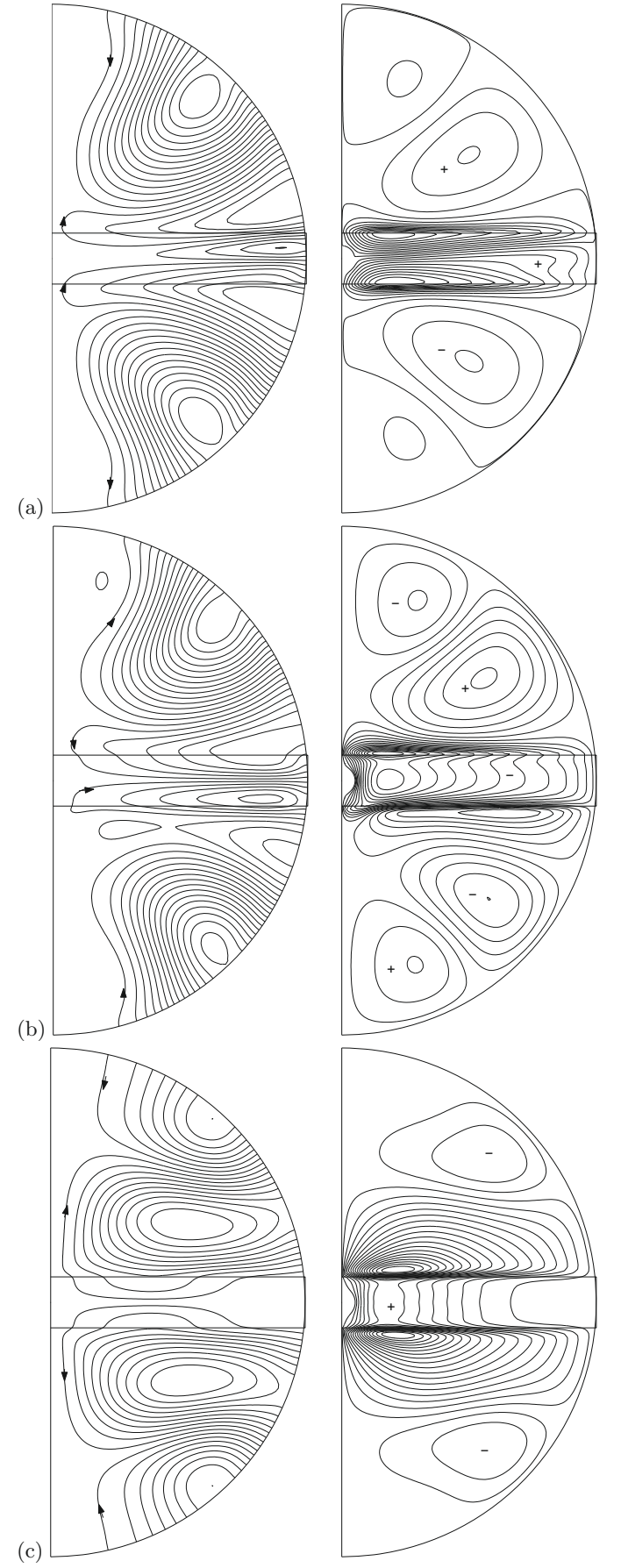

Fig. 4. Poloidal field line plots (left hand panels, cuts through one half of a meridian plane) and contours of toroidal field strength (right hand panels) for selected models with $R_{\alpha \text {, disc }}=20$. In the right hand panels regions of positive/negative toroidal field are indicated by $+/-$ signs respectively. Top row a), $C_{\text {wind }}=0, R_{\alpha \text {, halo }}=300$; row $\mathbf{b}$ ), $C_{\text {wind }}=100$, $R_{\alpha, \text { halo }}=300 ;$ row c), $C_{\text {wind }}=200, R_{\alpha \text {, halo }}=300$. Note the transition in field structure between rows $\mathbf{b}$ ) and $\mathbf{c}$ ) as $C_{\text {wind }}$ increases.

of the interstellar medium. In contrast, conventional mean-field galactic dynamos deal with an averaged wind velocity $u$. At the moment, there is no generally accepted method for converting $u$ to something resembling $U_{0}$, as used in our models. For orientation, note that with $\eta_{\text {disc }}=10^{26} \mathrm{~cm}^{2} \mathrm{~s}^{-1}, C_{\text {wind }}=100$ corresponds to $U_{0}=1.5 \mathrm{~km} \mathrm{~s}^{-1}$. We also note that Brandenburg et al. (1993) used a significantly larger value of $U_{0}$ when obtaining $\mathrm{X}$-shaped halo fields; the difference may be due to the detailed differences between the models, notably in the wind profile and rotation law, but also could be because quadrupole-like configurations appear less favourable for producing X-like structures (as discussed above), and so a larger value of $U_{0}$ is required. Further, and probably importantly, the Brandenburg et al. (1993) models had no $\alpha$-effect acting in the halo.

Detailed descriptions of the multiphase interstellar medium which include dynamo effects (e.g. Korpi et al. 1999) are still far from explaining global magnetic configurations. From a pragmatic viewpoint it would be sufficient to incorporate somehow the multiphase structure into a mean-field description, but the possibility is almost unexplored.

One more problem is that we have isolated a number of types of magnetic structures that can coexist in the disc and halo (disc enslaves halo, halo enslaves the disc, relatively independent generation etc). For one or two choices of parameters multiple (meta)stable solutions were found - e.g. when $R_{\alpha \text {, disc }}=20$, $R_{\alpha, \text { halo }}=400, C_{\text {wind }}=250, \eta_{\text {halo }} / \eta_{\text {disc }}=25$. We did not make a systematic search for such multiplicities, and it is probable that more could be found. Correspondingly, we may have missed solutions with $P_{\text {halo }}$ near $-1, P_{\text {disc }}$ near +1 even at parameters for which calculations were performed. We note that the various cases are not very well separated one from another, so various more-or-less exotic cases can occur at the transitions between the various types. A priori, there is nothing to rule out the occurrence of such field configurations in observed galaxies, although some may only occur in a very limited parameter range. Any progress in categorization here would be highly desirable. In the context of uncertainties connected with the form of the wind, we note also the possibility that galactic winds are likely to lack symmetry, either with respect to the disc plane or the rotation axis (or both). The consequences for the field geometry are uncertain and unexplored.

Further progress in the topic also requires a significant increase in the number of galaxies for which contributions to the polarized emission from disc and halo can be separated. We anticipate that new generation telescopes such as LOFAR and SKA will provide higher accuracy observational data on Faraday rotation and improve the situation.

Acknowledgements. D.S., R.B. and M.K. acknowledge financial support from the DFG-RFBR project 08-02-92881. The referee, Dr T. Konstantinos, is thanked for his detailed comments, which helped to improve the text.

\section{References}

Beck, R. 2009, ApSS, 320, 77

Beck, R., Brandenburg, A., Moss, D., Shukurov, A., \& Sokoloff, D. 1996 ARAA, 34, 155

Brandenburg, A., Donner, K. J., Moss, D., et al. 1992, A\&A, 259, 453

Brandenburg, A., Donner, K. J., Moss, D., et al. 1993, A\&A, 271, 36

Elstner, D., Meinel, R., \& Beck, R. 1992, A\&AS, 94, 587

Everett, J. E., Zweibel, E. G., Benjamin, R. A., et al. 2008, ApJ, 674, 258

Heesen, V., Beck, R., Krause, M., \& Dettmar, R.-J. 2009a, A\&A, 494, 563

Heesen, V., Krause, M., Beck, R., \& Dettmar, R.-J. 2009b, A\&A, 506, 1123

Kleorin, N., Moss, D., Rogachevskii, I., \& Sokoloff, D. 2002, A\&A, 387, 453

Kleorin, N., Moss, D., Rogachevskii, I., \& Sokoloff, D. 2003, A\&A, 400, 9

Korpi, M. J., Brandenburg, A., Shukurov, A., \& Tuominen, I. 1999, A\&A, 350, 230

Krause, M. 2009, Rev. Mex. Astron. Astrofis., 36, 25

Krause, M., Hummel, E., \& Beck, R. 1989, A\&A, 217, 4

Moss, D., \& Sokoloff, D. 2008, A\&A, 487, 197

Ruzmaikin, A., Shukurov, A., \& Sokoloff, D. 1988 (Dordrecht: Kluwer), Magnetic Fields of Galaxies

Sokoloff, D. D., \& Shukurov, A. M. 1990, Nature, 347, 51

Sun, X. H., Reich, W., Waelkens, A., \& Enßlin, T. A. 2008, A\&A, 477, 573 